\title{
An approach to design a quad-band NRI metamaterial for multipurpose sensor applications
}

\author{
Nipa Dhar ${ }^{1}$, Muhammad Asad Rahman ${ }^{1}$, and Md. Hossain ${ }^{1}$ \\ ${ }^{1}$ Chittagong University of Engineering and Technology
}

October 24, 2020

\begin{abstract}
The governing topic of this article is the design and investigation of a novel negative refractive index (NRI) metamaterial (MTM) in the microwave region. This multi-band MTM is realized by employing a square ring resonator along with two triangular-shaped resonators. Here, the designed unit cell produces double negative (DNG) regions to the extent of 3.6-3.9 $\mathrm{GHz}, 6.8-7.6 \mathrm{GHz}, 10.11-10.4 \mathrm{GHz}$, and 13.4-13.7 GHz and shows NRI properties in S-, C-, X-, and Ku-bands. Moreover, the design and performance evaluation of the $2 \times 2$ and $4 \times 4$ array configurations are investigated. Both of the structures create NRI band around S-, C-, X-, and Ku-bands. Also, the proposed MTM has an effective medium ratio of 13.8. The performance of the proposed MTM is validated by the sensor design study. Hence the proposed MTM is assigned for sensing pressure variation, for distinguishing used and unused transformer oil, and also to detect the several chemicals and chemical-water mixtures. The distinctive observation of the sensor study reveals $80 \mathrm{MHz}$ frequency difference between dark and clear oil and more than $340 \mathrm{MHz}$ resonance frequency shifting phenomena in X-bands for chemical sensing applications. Besides pressure sensor study exposes linear shifting of the resonances in S-, C-, X-, and Ku-bands.
\end{abstract}

\section{Hosted file}

Title page.pdf available at https://authorea.com/users/369901/articles/488716-an-approachto-design-a-quad-band-nri-metamaterial-for-multipurpose-sensor-applications

\section{Hosted file}

Manuscript.pdf available at https://authorea.com/users/369901/articles/488716-an-approachto-design-a-quad-band-nri-metamaterial-for-multipurpose-sensor-applications

\section{Hosted file}

Tables.pdf available at https://authorea.com/users/369901/articles/488716-an-approach-todesign-a-quad-band-nri-metamaterial-for-multipurpose-sensor-applications

\section{Hosted file}

Figures.pdf available at https://authorea.com/users/369901/articles/488716-an-approach-todesign-a-quad-band-nri-metamaterial-for-multipurpose-sensor-applications

\section{Hosted file}

Fig. 1(a).emf available at https://authorea.com/users/369901/articles/488716-an-approach-todesign-a-quad-band-nri-metamaterial-for-multipurpose-sensor-applications

\section{Hosted file}


Fig. 1(b).emf available at https://authorea.com/users/369901/articles/488716-an-approach-todesign-a-quad-band-nri-metamaterial-for-multipurpose-sensor-applications

\section{Hosted file}

Fig. 2.emf available at https://authorea.com/users/369901/articles/488716-an-approach-todesign-a-quad-band-nri-metamaterial-for-multipurpose-sensor-applications

\section{Hosted file}

Fig. 3(a).emf available at https://authorea.com/users/369901/articles/488716-an-approach-todesign-a-quad-band-nri-metamaterial-for-multipurpose-sensor-applications

\section{Hosted file}

Fig. 3(b). emf available at https://authorea.com/users/369901/articles/488716-an-approach-todesign-a-quad-band-nri-metamaterial-for-multipurpose-sensor-applications

\section{Hosted file}

Fig. 3(c).emf available at https://authorea.com/users/369901/articles/488716-an-approach-todesign-a-quad-band-nri-metamaterial-for-multipurpose-sensor-applications

\section{Hosted file}

Fig. 4(a).emf available at https://authorea.com/users/369901/articles/488716-an-approach-todesign-a-quad-band-nri-metamaterial-for-multipurpose-sensor-applications

\section{Hosted file}

Fig. 4(b).emf available at https://authorea.com/users/369901/articles/488716-an-approach-todesign-a-quad-band-nri-metamaterial-for-multipurpose-sensor-applications

\section{Hosted file}

Fig. 5(a).emf available at https://authorea.com/users/369901/articles/488716-an-approach-todesign-a-quad-band-nri-metamaterial-for-multipurpose-sensor-applications

\section{Hosted file}

Fig. 5(b). emf available at https://authorea.com/users/369901/articles/488716-an-approach-todesign-a-quad-band-nri-metamaterial-for-multipurpose-sensor-applications

\section{Hosted file}

Fig. 6(a).emf available at https://authorea.com/users/369901/articles/488716-an-approach-todesign-a-quad-band-nri-metamaterial-for-multipurpose-sensor-applications

\section{Hosted file}

Fig. 6(b).emf available at https://authorea.com/users/369901/articles/488716-an-approach-todesign-a-quad-band-nri-metamaterial-for-multipurpose-sensor-applications

\section{Hosted file}

Fig. 7(a).emf available at https://authorea.com/users/369901/articles/488716-an-approach-todesign-a-quad-band-nri-metamaterial-for-multipurpose-sensor-applications

\section{Hosted file}

Fig. 7(b) .emf available at https://authorea.com/users/369901/articles/488716-an-approach-todesign-a-quad-band-nri-metamaterial-for-multipurpose-sensor-applications

\section{Hosted file}


Fig. 8(a).emf available at https://authorea.com/users/369901/articles/488716-an-approach-todesign-a-quad-band-nri-metamaterial-for-multipurpose-sensor-applications

\section{Hosted file}

Fig. 8(b).emf available at https://authorea.com/users/369901/articles/488716-an-approach-todesign-a-quad-band-nri-metamaterial-for-multipurpose-sensor-applications

\section{Hosted file}

Fig. 8(c).emf available at https://authorea.com/users/369901/articles/488716-an-approach-todesign-a-quad-band-nri-metamaterial-for-multipurpose-sensor-applications

\section{Hosted file}

Fig. 8(d).emf available at https://authorea.com/users/369901/articles/488716-an-approach-todesign-a-quad-band-nri-metamaterial-for-multipurpose-sensor-applications

\section{Hosted file}

Fig. 9(a).emf available at https://authorea.com/users/369901/articles/488716-an-approach-todesign-a-quad-band-nri-metamaterial-for-multipurpose-sensor-applications

\section{Hosted file}

Fig. 9(b).emf available at https://authorea.com/users/369901/articles/488716-an-approach-todesign-a-quad-band-nri-metamaterial-for-multipurpose-sensor-applications

\section{Hosted file}

Fig. 9(c).emf available at https://authorea.com/users/369901/articles/488716-an-approach-todesign-a-quad-band-nri-metamaterial-for-multipurpose-sensor-applications

\section{Hosted file}

Fig. 10(a).emf available at https://authorea.com/users/369901/articles/488716-an-approachto-design-a-quad-band-nri-metamaterial-for-multipurpose-sensor-applications

\section{Hosted file}

Fig. 10(b).emf available at https://authorea.com/users/369901/articles/488716-an-approachto-design-a-quad-band-nri-metamaterial-for-multipurpose-sensor-applications

\section{Hosted file}

Fig. 11(a).emf available at https://authorea.com/users/369901/articles/488716-an-approachto-design-a-quad-band-nri-metamaterial-for-multipurpose-sensor-applications

\section{Hosted file}

Fig. 11(b).emf available at https://authorea.com/users/369901/articles/488716-an-approachto-design-a-quad-band-nri-metamaterial-for-multipurpose-sensor-applications

\section{Hosted file}

Fig. 11(c).emf available at https://authorea.com/users/369901/articles/488716-an-approachto-design-a-quad-band-nri-metamaterial-for-multipurpose-sensor-applications

\section{Hosted file}

Fig. 12(a).emf available at https://authorea.com/users/369901/articles/488716-an-approachto-design-a-quad-band-nri-metamaterial-for-multipurpose-sensor-applications

\section{Hosted file}


Fig. 12(b).emf available at https://authorea.com/users/369901/articles/488716-an-approachto-design-a-quad-band-nri-metamaterial-for-multipurpose-sensor-applications

\section{Hosted file}

Fig. 12(c).emf available at https://authorea.com/users/369901/articles/488716-an-approachto-design-a-quad-band-nri-metamaterial-for-multipurpose-sensor-applications

\section{Hosted file}

Fig. 13(a).emf available at https://authorea.com/users/369901/articles/488716-an-approachto-design-a-quad-band-nri-metamaterial-for-multipurpose-sensor-applications

\section{Hosted file}

Fig. 13(b).emf available at https://authorea.com/users/369901/articles/488716-an-approachto-design-a-quad-band-nri-metamaterial-for-multipurpose-sensor-applications

\section{Hosted file}

Fig. 14.emf available at https://authorea.com/users/369901/articles/488716-an-approach-todesign-a-quad-band-nri-metamaterial-for-multipurpose-sensor-applications

\section{Hosted file}

Fig.15(a).emf available at https://authorea.com/users/369901/articles/488716-an-approach-todesign-a-quad-band-nri-metamaterial-for-multipurpose-sensor-applications

\section{Hosted file}

Fig.15(b) .emf available at https://authorea.com/users/369901/articles/488716-an-approach-todesign-a-quad-band-nri-metamaterial-for-multipurpose-sensor-applications

\section{Hosted file}

Fig. 16.emf available at https://authorea.com/users/369901/articles/488716-an-approach-todesign-a-quad-band-nri-metamaterial-for-multipurpose-sensor-applications

\section{Hosted file}

Fig. 17.emf available at https://authorea.com/users/369901/articles/488716-an-approach-todesign-a-quad-band-nri-metamaterial-for-multipurpose-sensor-applications

\section{Hosted file}

Fig. 18(a).emf available at https://authorea.com/users/369901/articles/488716-an-approachto-design-a-quad-band-nri-metamaterial-for-multipurpose-sensor-applications

\section{Hosted file}

Fig. 18(b).emf available at https://authorea.com/users/369901/articles/488716-an-approachto-design-a-quad-band-nri-metamaterial-for-multipurpose-sensor-applications

\section{Hosted file}

Fig. 19(a).emf available at https://authorea.com/users/369901/articles/488716-an-approachto-design-a-quad-band-nri-metamaterial-for-multipurpose-sensor-applications

\section{Hosted file}

Fig. 19(b).emf available at https://authorea.com/users/369901/articles/488716-an-approachto-design-a-quad-band-nri-metamaterial-for-multipurpose-sensor-applications

\section{Hosted file}


Fig. 20(a).emf available at https://authorea.com/users/369901/articles/488716-an-approachto-design-a-quad-band-nri-metamaterial-for-multipurpose-sensor-applications

\section{Hosted file}

Fig. 20(b).emf available at https://authorea.com/users/369901/articles/488716-an-approachto-design-a-quad-band-nri-metamaterial-for-multipurpose-sensor-applications

\section{Hosted file}

Fig. 21(a).emf available at https://authorea.com/users/369901/articles/488716-an-approachto-design-a-quad-band-nri-metamaterial-for-multipurpose-sensor-applications

\section{Hosted file}

Fig. 21(b).emf available at https://authorea.com/users/369901/articles/488716-an-approachto-design-a-quad-band-nri-metamaterial-for-multipurpose-sensor-applications

\section{Hosted file}

Fig. 21(c).emf available at https://authorea.com/users/369901/articles/488716-an-approachto-design-a-quad-band-nri-metamaterial-for-multipurpose-sensor-applications

\section{Hosted file}

Fig. 22(a).emf available at https://authorea.com/users/369901/articles/488716-an-approachto-design-a-quad-band-nri-metamaterial-for-multipurpose-sensor-applications

\section{Hosted file}

Fig. 22(b).emf available at https://authorea.com/users/369901/articles/488716-an-approachto-design-a-quad-band-nri-metamaterial-for-multipurpose-sensor-applications

\section{Hosted file}

Fig. 22(c).emf available at https://authorea.com/users/369901/articles/488716-an-approachto-design-a-quad-band-nri-metamaterial-for-multipurpose-sensor-applications

\section{Hosted file}

Fig. 22(d).emf available at https://authorea.com/users/369901/articles/488716-an-approachto-design-a-quad-band-nri-metamaterial-for-multipurpose-sensor-applications

\section{Hosted file}

Fig. 23.emf available at https://authorea.com/users/369901/articles/488716-an-approach-todesign-a-quad-band-nri-metamaterial-for-multipurpose-sensor-applications 